\title{
AN EVALUATIVE REVIEW OF SIMULATED DYNAMIC SMART 3D OBJECTS.
}

\author{
Romeijn, H. ${ }^{1}$, Sheth, F. ${ }^{1}$, Pettit, C.J. ${ }^{2}$ \\ ${ }^{1}$ Future Farming Systems Research Division, Department of Primary Industries, Parkville, Victoria, Australia - \\ harmen.romeijn@dpi.vic.gov.au, falak.sheth@dpi.vic.gov.au \\ ${ }^{2}$ Faculty of Architecture, Building and Planning, The University of Melbourne, Parkville, Victoria, Australia - \\ cpettit@unimelb.edu.au
}

KEY WORDS: Modelling, Visualisation, Object, Three-dimensional, Comparison, Software

\begin{abstract}
:
Three-dimensional (3D) modelling of plants can be an asset for creating agricultural based visualisation products. The continuum of $3 \mathrm{D}$ plants models ranges from static to dynamic objects, also known as smart 3D objects. There is an increasing requirement for smarter simulated 3D objects that are attributed mathematically and/or from biological inputs. A systematic approach to plant simulation offers significant advantages to applications in agricultural research, particularly in simulating plant behaviour and the influences of external environmental factors. This approach of 3D plant object visualisation is primarily evident from the visualisation of plants using photographed billboarded images, to more advanced procedural models that come closer to simulating realistic virtual plants. However, few programs model physical reactions of plants to external factors and even fewer are able to grow plants based on mathematical and/or biological parameters. In this paper, we undertake an evaluation of plant-based object simulation programs currently available, with a focus upon the components and techniques involved in producing these objects. Through an analytical review process we consider the strengths and weaknesses of several program packages, the features and use of these programs and the possible opportunities in deploying these for creating smart 3D plant-based objects to support agricultural research and natural resource management. In creating smart 3D objects the model needs to be informed by both plant physiology and phenology. Expert knowledge will frame the parameters and procedures that will attribute the object and allow the simulation of dynamic virtual plants. Ultimately, biologically smart 3D virtual plants that react to changes within an environment could be an effective medium to visually represent landscapes and communicate land management scenarios and practices to planners and decision-makers.
\end{abstract}

\section{INTRODUCTION}

3D objects can be defined as visual representations of the elements that comprise real and fictitious geographies (Pettit \& $\mathrm{Wu}, 2008$ ). Pettit et. al., (2009) further expands upon this definition of $3 \mathrm{D}$ objects by describing them as visual representations of flora, fauna, built infrastructure and people. Flora based objects particularly represent a challenge within object modelling to effectively capture the dynamic physiological nature of plants. The models may be static, capturing plant form at a particular point in time, or developmental, describing the form as a result of growth (Prusinkiewicz, 2004). Static depictions have been investigated in multiple techniques and underlying architectures formalised over a range of methods (Godin, 2000; de Visser et. al., 2002; Prusinkiewicz \& Lindenmayer, 2004; Deussen \& Lintermann, 2005). In recent years, with the advent of advanced and powerful computing and modelling techniques, more development of functional plant models/growth models have become available, which consider many interacting processes by combining the modelling of physiological processes with the architecture of the plant (Sarlikioti et. al., 2010; Fourcaud et. al., 2008; Vos et. al., 2010). Modelling growth has become key research activity within the fields of agriculture, forestry and environmental sciences (Godin \& Sinoquet, 2005; Fourcaud $e$ t. al., 2008). These new growth-modelling tools allow us to increase our understanding of plant form development in relation to its environment (de Visser et. al., 2002).

This paper provides a broad review of the best available tools according to their use and output. There are two main objectives addressed in the body of this paper. Firstly, a literature and software review of a selection of existing plantbased simulation platforms was undertaken. The selection of programs to be assessed has been primarily based upon the differing ranges of systems used to generate plant-based models. Whilst this is not an exhaustive list, it does detail an assortment of available programs and methodologies. Within this review, the evaluation focuses both on the systems involved in producing plant-based objects and also program options to further create and develop plant models. By means of this review, the relative strengths and weaknesses of each of the programs has been addressed.

Secondly, an assessment on which software tools can effectively simulate a plant-based object as accurately as possible has been done. This has been directly informed from the results of the abovementioned review. To this purpose, plant-based model outputs from a selection of the reviewed software packages have been evaluated and compared against a set of criteria; computing requirements, model display and detail and end use. Any such plant object will need to, as accurately as possible, model the physiological development of a plant and the interactions a plant has with its environment, whilst also retaining a sense of realism.

\section{REVIEW OF PLANT-BASED SIMULATION SOFTWARE PLATFORMS}

\subsection{Plant-based object generation methods}

For over thirty years, botanists and computer scientists have made considerable efforts to develop effective methods to synthetically generate natural objects (Deussen \& Lintermann, 2005). The design and creation of natural plant-based objects has evolved since original computer branching simulations. Primary uses of simulated plants are within the games and 
movie industries; however, there has been a progression within empirical based scientific research to utilise computer generated plants for genetic, physiological and environmental simulations. In use today there are three primary approaches to plant-based visualisation. As described in Pettit et. al., (2009), these principal methods are image based models, billboard clouds and 3D models.

Image based models: Often referred to as billboards, this is a simple method that can be relatively effective under certain circumstances. As shown to the left in Figure 1 this method uses an image of an object, which is applied to either one or two rectangular polygons. They can only be made as static images; plant related detail cannot be attributed within the image. Image based models are best suited for simple landscape visualisations where detail is not required as they are only a representation of a plant.

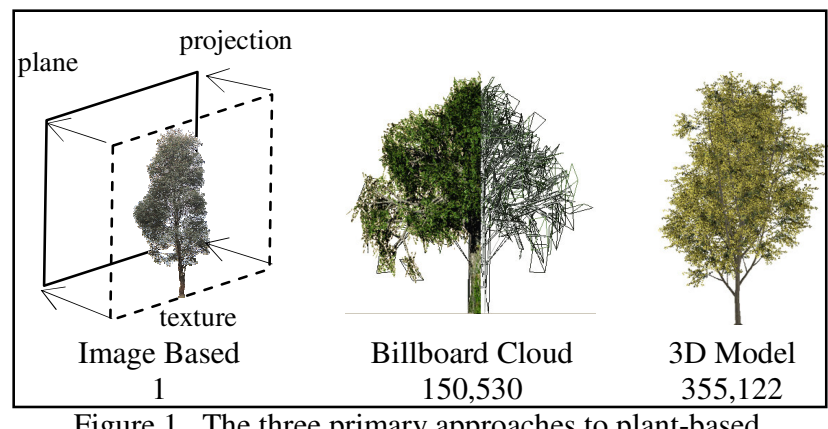

Figure 1. The three primary approaches to plant-based visualisation. The bottom numbers represent the numbers of polygons used to create each model.

Billboard clouds: Billboard clouds are a continuation of the image based model. The billboard cloud approach decomposes the original object into subsets of patches and replaces each subset by a billboard, these billboards are fixed in position, in relation to their location on the plant, and the final image is the composition of their images (Garcia et. al., 2005). As shown in the centre of Figure 1, billboard clouds provide a higher level of detail compared to image based models. The level of realism depends on the amount of polygons used to construct the object. However, as with image based models, objects described as billboard clouds are static, and the dynamic nature of plantbased objects cannot be expressed.

3D Models: When describing 3D models, there are several methods that exist for the purpose of creating an object, each with associated levels of detail. Plants can be detailed at a basic level using primitive geometric 3D shapes. Rendering of these shapes to detail textures, leaves, branches and shadows can help define these basic plant shapes. This can be limited to formal or manicured plants, such as hedges or pruned shrubs. Limitations can arise when trying to define every small twig, which can be very intensive for what is meant to be a simple model.

3D models are referred to as geometric models, which are primarily concerned with depicting the formal structure and architecture of a plant. As shown to the right of Figure 1, objects built using geometric models can be relatively complex, but simply described through a few basic, yet powerful, algorithms. Depending on the skill of the operator, some pure mathematical algorithms as fractals, binary trees and particle systems, can give birth to attractive shapes of plants (de Reffye $\& \mathrm{Hu}, 2003)$. Deussen and Lintermann (2005) expand upon the underlying characteristics of geometric models by introducing the ideas of Procedural and Rule-based Models. Procedural models are described as parameterised algorithms that are designed for the production of a certain type of plant. Whereas rule-based models are based upon rule systems, which produces from a simple initial state a complex final state.

Procedural methods are more associated with particular plant examples with the parameters that define the object are inbuilt into the system. The recursive nature of plants can be linked to aspects of fractal creation, and through the inclusion of parameters and limits, complex tree branching structures can be generated. An algorithm produced by Oppenheimer (1986), demonstrates this recursive fractal nature of trees. Also introduced is the idea of randomness, which alleviates selfsimilarity that can be seen in the object at each level. This method is recursive; it generates a trunk which leads to large branches and so on until twigs are simulated.

Rule-based models share the same principals and underlying complexity as procedural models, but they are more general in nature. However, they are able to produce intricate and diverse plant structures. Lindenmayer systems, or L-Systems, are an example of rule-based models (Lindenmayer, 1968). L-Systems are a mathematical tool to model plant development and structure that are derived from formal grammars for string generation (de Visser et. al., 2002). The principles of geometric patterns and repetition are a recurring theme in the plant world; it is possible to use mathematical means to describe these patterns. The basic language and grammar used by an LSystem can be easily displayed in a graphical format, and new dimensions can be added to reproduce 3D images.

The strength behind procedural and rule-based models is that from a set of primary algorithms, or rules, a large and complex object can be created. What is more, these geometric models can be combined with process-based models to create more complex and realistic objects. Process-based models, which are more associated with plant structures, are mathematical models based on the depiction of basic physiological aspects of plant growth, such as photosynthesis, nutrient cycles and climatic effects. Process-based models take into account, at the physiological scale, plant responses to particular site-based factors. The formation of an effective process-based model involves two tasks; identify the processes that express the physiological and phenological actions of the plant and then establish values for the model that describe these actions.

Unlike image based models and billboard clouds, 3D methods are able to simulate both the visual and the dynamic nature of plants. These methods can be developed further to generate more detailed models that are structurally correct as well as biologically attributed. Through the combination of geometric and process-based models, a functional-structural plant model can be created. The principles and exploration behind these models are beginning to be detailed in the literature (Godin \& Sinoquet, 2005; Vos et. al., 2010) with applications seen within scientific research (Sarlikioti et. al., 2010). Models and platforms are also being designed around these principles (de Reffye \& Hu, 2003; Barczi et. al., 2007).

\subsection{Review method}

From an agricultural systems research perspective, an analytical review on the capabilities and functionality of several plantbased object simulation packages has been undertaken. This assessment will firstly consider the perceived strengths and weakness within the selected range of products and then test the functionality of each program. 
The breadth of plant object visualisation software platforms that are available is extensive. Plant objects that are generated can range from the simple imaged based models to detailed geometric models, and the levels of control into this associated detail can vary between programs. Programs that have been selected provide a general cross-section of available programs and are principally centred on the three-dimensional aspects of plant modelling. These programs represent an assortment of available packages and end-uses. Seven plant object creation software packages have been chosen for this review; Arbaro, XFrog, PlantStudio, L-Studio, GreenLab, SpeedTree and natFX

Each of the selected software programs contains strengths and weaknesses that can either relate to the internal functionality or the external usability of the program. Strengths refer to the characteristics of the program that give it an advantage over others, whereas weaknesses refer to characteristics that disadvantage the program. These strengths and weaknesses can be compared, as seen in Table 1, to assess the systems involved in producing plant-based objects and program options to further create and develop plant models. The factors presented are not an exhaustive listing, but they provide a range perceived strengths and weaknesses based on a research perspective.

Through this, the general functionality of each plant-based software package can be evaluated through a set of criteria which encompass three general themes; hardware and software requirements, model display capacity and model end use. From an agricultural research and natural resource management sphere the main assessment point will centre upon questioning what packages can effectively simulate plant-based objects accurately.

The methodology used for the initial strength and weakness analysis is direct user interaction (Kujala, 2003), where the overall useability of the program is assessed. Similarly, methodologies for functionality testing are done through direct user interaction, but these are limited in scope to the outlined set of criteria. Hence, results that are discussed are subjective and can be biased both towards the users experience with the program and the research based perspective.

\subsection{Program capabilities}

Across the array of programs, with the exception of PlantStudio, realistic plant-based models can be generated. The realism seen in the majority of these programs is at a visual level; i.e. objects appear correct in their architecture and appearance. Alternately, GreenLab produces models that may lack a realistic appearance, but the architecture and structure of an object is correct. More so, simulated models are considered realistic in terms of the 'correctness' of the model, in that they can be attributed with and produce biological based information. This biological correctness is also seen in L-Studio. These two programs are research orientated in their design and outputs, differing from other packages that are more industry based with outputs used for games, movies and other such uses. The research orientated scope of GreenLab and L-Studio further strengthens the accuracy of the model by developing models based on botanical knowledge and technical expertise.

Visual realism can be enhanced in several of the packages through rendering options. These options can be inbuilt into the program, such as through colour palette selection or texturebased inputs from images. Alternatively, some programs allow for the ability to export the model to secondary programs for rendering purposes.
For the enhancement of the structural components, or the biological accuracy of an object, the majority of programs have options that model different dynamic features of a plant. These dynamic options range from external stimuli interactions to internal physiological processes. Dynamic functions that relate to external factors include physical interactions with other objects and wind effects, as seen in SpeedTree, natFX and others. However, these types of dynamic options only affect the structure of a plant object based on computer modelling and can lack biological fidelity. Some of the dynamic options available, such as those in natFX, include time based functions plant age or seasonal variation can be visualised. Further to this, other programs such as XFrog and PlantStudio can model growth of a plant over a set time period. Nevertheless, these growth animations and time functions are based on mathematical equations and modelling and can be deficient from a biological perspective. GreenLab and L-Studio allows for the dynamic modelling of interactions a plant may have based on environmental stimuli, which include temperature, light and water responses. These dynamic options, unlike others in other programs, are informed though correct real-world information.

\begin{tabular}{|c|ccccccc|}
\hline Strengths & $\mathbf{1}$ & $\mathbf{2}$ & $\mathbf{3}$ & $\mathbf{4}$ & $\mathbf{5}$ & $\mathbf{6}$ & $\mathbf{7}$ \\
\hline Realistic & $\mathbf{X}$ & $\mathbf{X}$ & & $\mathbf{X}$ & $\mathbf{X}$ & $\mathbf{X}$ & $\mathbf{X}$ \\
\hline Dynamic & & $\mathbf{X}$ & $\mathbf{X}$ & $\mathbf{X}$ & $\mathbf{X}$ & $\mathbf{X}$ & $\mathbf{X}$ \\
\hline Inbuilt rendering & $\mathbf{X}$ & $\mathbf{X}$ & $\mathbf{X}$ & $\mathbf{X}$ & & $\mathbf{X}$ & $\mathbf{X}$ \\
\hline Export options & $\mathbf{X}$ & $\mathbf{X}$ & $\mathbf{X}$ & $\mathbf{X}$ & & $\mathbf{X}$ & $\mathbf{X}$ \\
\hline User base/support & & $\mathbf{X}$ & & & & $\mathbf{X}$ & $\mathbf{X}$ \\
\hline Plant library & & $\mathbf{X}$ & & & & $\mathbf{X}$ & $\mathbf{X}$ \\
\hline Simple to use & $\mathbf{X}$ & & $\mathbf{X}$ & & & & \\
\hline Open source & $\mathbf{X}$ & & $\mathbf{X}$ & & $\mathbf{X}$ & & \\
\hline Generate all plant types & & $\mathbf{X}$ & & $\mathbf{X}$ & $\mathbf{X}$ & $\mathbf{X}$ & $\mathbf{X}$ \\
\hline Research orientated & & & & $\mathbf{X}$ & $\mathbf{X}$ & & \\
\hline Generate new plants & $\mathbf{X}$ & $\mathbf{X}$ & $\mathbf{X}$ & $\mathbf{X}$ & $\mathbf{X}$ & $\mathbf{X}$ & \\
\hline Weaknesses & & & & & & & \\
\hline Limited to tree models & $\mathbf{X}$ & & & & & & \\
\hline Herbaceous plants only & & & $\mathbf{X}$ & & & & \\
\hline Limited rendering & & & $\mathbf{X}$ & & & & \\
\hline Limited export options & & & & $\mathbf{X}$ & $\mathbf{X}$ & & \\
\hline No assured updates & $\mathbf{X}$ & & $\mathbf{X}$ & $\mathbf{X}$ & $\mathbf{X}$ & & \\
\hline No plant library & $\mathbf{X}$ & & $\mathbf{X}$ & $\mathbf{X}$ & $\mathbf{X}$ & & \\
\hline Complex to use & & & & $\mathbf{X}$ & $\mathbf{X}$ & & \\
\hline Proprietary & & $\mathbf{X}$ & & $\mathbf{X}$ & & $\mathbf{X}$ & $\mathbf{X}$ \\
\hline Costs with program & & $\mathbf{X}$ & & $\mathbf{X}$ & & $\mathbf{X}$ & $\mathbf{X}$ \\
\hline Costs with plant library & & $\mathbf{X}$ & & & & $\mathbf{X}$ & $\mathbf{X}$ \\
\hline Generate plants from 'seed' & & & & & & & $\mathbf{X}$ \\
\hline
\end{tabular}

Table 1. Strengths and weaknesses analysis table. (1.Arbaro, 2.XFrog, 3.PlantStudio, 4.L-Studio, 5.GreenLab, 6.SpeedTree, 7.natFX)

The nature of the programs external capabilities can affect the overall use and experience that may occur with a program. Open source or open licence programs, such as Arbaro or GreenLab, can be attractive to use as they are free for research purposes. Also, there is the possibility for the user to develop or modify the program to their needs, but this depends on the technical expertise of a user and the complexity behind the programs language structure. However, these programs can lack technical support from the developer. Also they can have a low user base, which can mean community support and knowledge will be minimal.

The costs for proprietary programs can vary in price. XFrog is one of the lower priced programs; the stand-alone package is up to $\$ 300$. SpeedTree has the highest cost amongst the programs within this evaluation of over $\$ 12,000$, which can render it 
unobtainable from an academic or research standpoint. Proprietary programs are fixed in their development stage and there is reliance on the software company to develop and upgrade the program. But the proprietary software packages have technical support and a large user base to assist in troubleshooting. The research based programs of GreenLab and LStudio have a different user base based on academics, researchers and institutions and from these sources, information about the program and its use can be gained.

All programs have a few example models included in the installation, which can range from a single object to over 20 differing plant objects. Included with the proprietary software packages, is the availability of plant model libraries. These libraries can range in size from large selections above 450 objects, as seen with natFX, to a comparatively small library of about 150 objects seen with SpeedTree. The plant objects in the libraries do have associated costs; a single plant in natFX and SpeedTree can cost $\$ 40$ and $\$ 50$ respectively, which can increase outlay costs when working with these programs. There exist bundle options where multiple plants can be purchased at a reduced price. XFrog has this alternative where an agricultural plant bundle of 20 plant types costs $\$ 200$, so a single plant costs $\$ 10$, which can make this program more attainable cost-wise.

All programs allow the user to generate a plant-based model and/or modify existing models. However, natFX is limited in this sense; a plant needs to be purchased from their library, which then can be modified, there is no ability to generate a new plant model. The relative learning curve of each program is very easy and most programs are intuitive in their use. Most have text entry boxes, sliders and help pop-ups that guide their use. Exceptions to this are the research orientated programs of GreenLab and L-Studio. A degree of botanical knowledge is needed to understand the nomenclature used in these programs. However, since they have a plant-based research focus, potential users can have an understanding of the terminology.

\subsection{Program functionality}

Hardware and Software: Of the evaluated products, most act as a stand-alone product. Two programs, natFX and GreenLab are 'plug-ins' and require a secondary program to function. Several of the programs are cross-platform software, they are able to run across Windows, Macintosh and Linux based operating systems. Some, such as L-Studio, are limited to Windows based operating systems. Both SpeedTree and natFX have the highest hardware requirements and therefore can be strongly influenced by the computers processing speed, video card and available RAM memory.

The proprietary programs are polished in terms of user interfaces, and the use of these programs is aided by graphical window displays, help pop-ups and guides. GreenLab and LStudio, due to its research based orientation, can be complex to correctly use and some terminology used in the interface of the program needs to be comprehended initially before use.

Model Display: The realism in the models produced by the software packages can differ based on structural or biological correctness. GreenLab, being a research orientated program, produces models that are not wholly realistic. However, models produced can be biologically correct. The functional-structural nature of the program creates models that can be both correct in its physiological form and phenological attributes, but visually it lacks realism. XFrog, conversely, produces models that are visually correct and realistic, but with little biological foundation. Models that are created can have the correct physiological form, but phenological functions cannot be attributed to the model. SpeedTree and natFX, in a manner, produce models that can be realistic visually and also have some phenological attributes associated with it, such as wind effects, seasonality and growth age. But these attributes are not biologically correct; they are more a result of modelling interactions generated by the program.

The realism of the models created in each program is a function of the detail that is inherent in the object. Models are created through the combination of multiple triangles, or polygons, that are joined to produce a surface. These can be flat so as to form leaves, or shaped into geometric forms to simulate trunks, branches or other structures. In models created by some programs, such as Plant Studio or GreenLab, these elements cannot be directly modified; they are set for each model once generated. Models are also fully geometric in design; billboards are not used in the creation of an object. This is also true for other programs, such as Arbaro and XFrog, where models are constructed from geometric shapes. Using XFrog, for example, an object is formed through a hierarchy of primitives, the geometric shapes, and components, the structural formations found within plants, i.e. trunks and leaves. Within natFX there is a range of options available to display an object. Models can either be a full geometry plant, a billboard cloud, an image based model, or a hybrid comprising elements of all three methods.

The level of detail (LOD) in an object, that is its complexity based on the number of polygon faces, can vary largely between each program. Using a wheat plant model as an example (Figure 2), XFrog at a low LOD uses about 484 polygons, at the highest LOD there are 26,887 polygons used per wheat stalk. Arbaro is similar in the LOD used in its wheat model at 25,202 polygons. GreenLab, with the same model, is similar to XFrog at it lowest LOD with 672 polygons. However, the LOD in GreenLab cannot be changed directly; it is set for an object once generated, but polygon counts are always low for an object. This can also be seen when comparing a tomato model between GreenLab and XFrog. In GreenLab the LOD is low at 6,876 polygons, in XFrog the lowest LOD is 91,140 polygons.

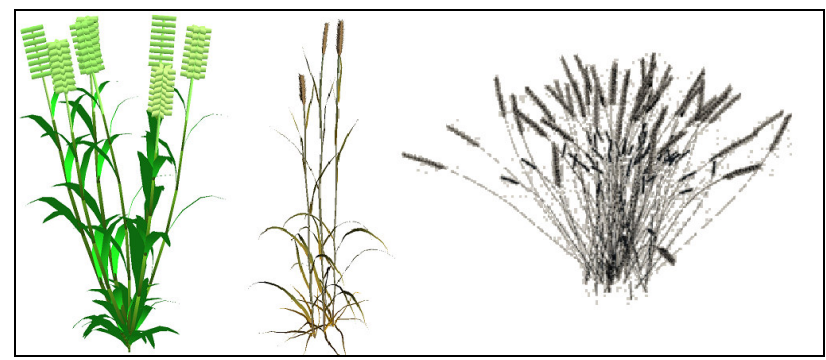

Figure 2. Comparison of three wheat object models. GreenLab (left), XFrog (centre), Arbaro (right).

Similarly, when comparing a tree-based object between SpeedTree, XFrog and natFX (Figure 3), there can be a large variation in LOD and polygon counts. SpeedTree at a low LOD uses 6,145 polygons for a tree and a high LOD uses 318,848 polygons. XFrog has the largest variation from a low LOD of 46,471 polygons for a tree up to $3,918,324$ polygons for a high LOD tree object. The range of model generation methods in natFX allows for a large control on the LOD. A tree image based model has a low LOD at 4 polygons, whereas a full geometry model of the same tree with a high LOD has 199,487 polygons. 


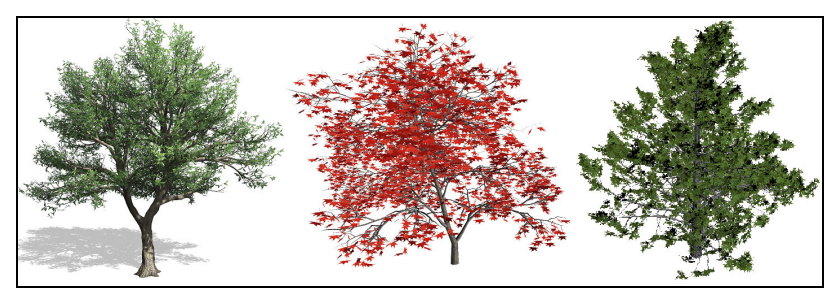

Figure 3. Comparison of three tree object models. SpeedTree (left), XFrog (centre), natFX (right).

Apart from Arbaro, each program possesses particular dynamic functions and interactions. As mentioned, natFX is able to simulate growth, seasons and wind interactions. It also is able to provide animations of growth both for differing ages of a plant or seasons, albeit this animation is based upon modelling parameters rather than biological constraints. SpeedTree has wind effect options and range of real world physics, such as plant breakages and force interactions. PlantStudio has options to breed plants over multiple generations, this allows for unique plants to be generated from a parent object. PlantStudio also has a growth simulator. However, as with natFX, growth is defined by a logarithmic function, which determines plant biomass at a particular stage in the function. XFrog also possesses an array of dynamic options such as wind affects and also growth animations, which are based on the underlying procedures in each component. These can be set for a particular time and by allowing the component procedures to initiate at a set time, animations of growth can be built. L-Studio and GreenLab differ from these other two programs in that dynamic options are based upon environmental factors. This includes factors that may influence plant growth such as temperature, water or light. These dynamic options are modelled based on biological factors rather than modelled and interpolated through mathematical means.

Use of Model: All the software programs have a degree of portability, both in terms of exporting or importing. Options exist within XFrog for exporting to 3D file formats such as CAD, Wavefront and VRML, as well as standard 2D formats such as png, bitmap and jpeg. This is also true for others where a large array of export file formats is present with the Wavefront file format existing through the majority of programs. GreenLab is the most limiting in terms of exporting and importing options. GreenLab works with its own internal file formats, and this limits the use of 3D models outside the program. However, there is ability to export models to $2 \mathrm{D}$ formats such as gif and bitmap.

File sizes, using the Wavefront file format as an example, varies dependent on aspects like the polygon count in the object and the compression ratio of the program. A broad leaf tree in SpeedTree can range from $10 \mathrm{Mb}$ in size for a low resolution object up to $60 \mathrm{Mb}$ for a high resolution plant. XFrog has the largest variance in Wavefront file sizes, from $6 \mathrm{Mb}$ for a low detail broad leaf tree up to $410 \mathrm{Mb}$ for a tree in the highest resolution settings. Alternately, natFX has a range of options for exporting to Wavefront from image based models to full geometry objects; hence file sizes can range from $1 \mathrm{~Kb}$ to $25 \mathrm{Mb}$ for a broad leaf tree. The large variations in file sizes can limit the application of these programs, particularly if a forest plantation or a food crop visualisation is to be simulated. If a full resolution large file size object is used then there can be limits on the amount of space each object utilises and also the computers system memory that can be used in simulating multiple objects.
Import options are available over most programs, primarily the proprietary programs. These inputs include $2 \mathrm{D}$ image formats which can be used for texture related inputs into models. The importing of full 3D plant models is also available over multiple formats, such as Wavefront and Autodesk FBX, in the proprietary programs, albeit in most cases these are treated as non-plant objects and can not be modified by the tools within a specific program. This direct compatibility of file formats between varying programs can allow for rendering of objects to improve visual aspects. But this has limited use in research based applications. This is due to the fact that these imported objects are static in nature and no modifications or additions can be made and no information can be gained from them.

Model openness refers to how customisable a model is within a program. This is seen in most programs where objects can be created from a set of geometric shapes. natFX is limited in its model openness; objects cannot be created from the base up. Existing plants can be modified based on initial parameters, but it can be limiting in that a tree cannot be changed into grass. Some, such as PlantStudio, have automatic plant creation methods where objects can be generated, but established models can't be changed. Objects in Arbaro can be edited within the programs graphical interface, or the object files can be edited directly in a text editor. This duality in object editing methods is also seen in GreenLab and L-Studio. However, this level of customising does require an intrinsic understanding of the programs format and how it reads these files and the value within.

The majority of software programs are able to generate objects for use in virtual terrain settings and digital globes. The Wavefront file format, for example, can be used within Google applications like Google Earth. SpeedTree has a world builder feature where Collada, Autodesk or Wavefront files for terrain and plant objects can be used to create a virtual world and then exported to be used in other digital globe or virtual world applications. L-Studio and GreenLab lack this functionality, objects created are only used within the program itself and no direct method exists to use a plant model in a virtual environment. The main end use application behind most of these mentioned programs is to create plants for use in applications such as games, architectural design or planning purposes. This can reduce the functionality of these programs when applying within a research based focus. These programs are able to visually represent a tree plantation or food crop, but no information can be gained directly from this type of scene apart from a visual based analysis. The programs that can create objects that are dynamic to their environment and attributed with biological information cannot be used effectively in a virtual terrain setting.

\section{CONCLUSION}

This review of plant-based object modelling software allows for the opportunity to develop an exemplar model, which can potentially communicate agricultural based scenarios and practices as well as provide information upon plant growth and productivity based on biological inputs. If an object is to be used for research orientated purposes, then visual correctness is not a priority, rather the characteristics and interactions the object contains is of importance. However, if an object is used to inform planning and management actions, such as those from an urban or rural planning point of view, then visual representations can become important, as well as the dynamic connections it has in its environment. 
From the analysis of the range of programs it is noted that not one program is wholly comprehensive in terms of combining realism and dynamic features to simulate a biologically correct and environmentally reactive plant object. The majority contain components that are able to simulate dynamic interactions or visually represent plants, but these are spread across each program. By using XFrog and natFX as examples, they are able to visually represent an object in a physiologically correct sense. Also present are options to model dynamic interactions, albeit through mathematical procedures rather than informed by biological associations. Through comparisons of these programs against GreenLab, there is a focus on producing objects that are biologically correct, with objects that are attributed by biological parameters that inform a correct physiological shape and phenological processes. This creates a plant object that can be shaped by dynamic environmental interactions and report on such attributes such as plant biomass. However, final models lack visual detail and may not translate to uses outside a research sphere.

To create an exemplar model, certain gaps and priorities need to be identified. From an agricultural research focus, physiological parameters that will inform both plant architecture and plant processes are necessary. To determine correct architecture, measurements such as stem/trunk diameter, branch length and leaf sizes will need to recorded and used to inform the object. Correct visual representations of the plant will require input images of the bark, leaves and flowers. More so important is how the plant in question interacts within its environment (temporal dynamics). Relations and processes like growth rates, water requirements, optimal temperatures, resource competition and light interception are important parameters. This collation of knowledge requires the combination of botanical based information and computer modelling approaches, which is already seen in the simulation of objects in GreenLab.

The functional-structural approach, as used within GreenLab, can be seen to be the most advantageous direction with the best opportunities for research orientated objectives. There are limitations within such programs, which are more related to visual representations of the plant. However, for agricultural research purposes, these visual limitations are less significant. It is the underlying processes within the plant, factors such as growth rates and the final output which are of concern. For simply visualising a plant in a virtual terrain, then programs such as SpeedTree or XFrog can be utilised. To truly have a visually correct and reactive plant that changes to stimuli in the virtual environment then a combination of programs, or the further development of those in use, is necessary.

\section{REFERENCES}

Barczi, J.F., Rey, H., Caraglio, Y., De Reffye, P., Barthelemy, D., Dong, Q. X., and Fourcaud, T. 2007. AmapSim: A structural whole-plant simulator based on botanical knowledge and designed to host external functional models. Annals of Botany, 101(8), pp. 1125-1138.

Deussen, O., and Lintermann, B. 2005. Digital Design of Nature. Springer Publishing. Berlin,

Fourcaud, T., Zhnag, X., Stokes, A., Lambers, H., and Korner, C. 2008. Plant growth modelling and applications: The increasing importance of plant architecture in growth models. Annals of Botany, 101, pp. 1053-1063.

Garcia, I., Sbert, M., and Szirmay-Kalos, L. 2005. Tree Rendering with Billboard Clouds. Third Hungarian Conference on Computer Graphics and Geometry Proceedings, Budapest, 2005, pp.9-15.

Godin, C. 2000. Representing and encoding plant architecture: A review. Annals of Forest Science, 57, pp. 413-438.

Godin, C., and Sinoquet, H. 2005. Functional-structural plant modelling. New Phytologist. 166, pp. 705-708.

Kujala, S. 2003. User involvement: A review of the benefits and challenges. Behaviour and Information Technology, 22(1), pp. 1-16.

Lindenmayer, A. 1968. Mathematical models for cellular interaction in development, Parts I and II. Journal of Theoretical Biology, 18, pp. 280-315.

Oppenheimer, P. 1986. Real time design and animation of fractal plants and trees. SIGGRAPH ' 86 Proceedings of the $13^{\text {th }}$ Annual Conference on Computer Graphics and Interactive Techniques, Dallas, 1986, pp. 55-64.

Pettit, C.J., and Wu, Y. 2008. A Virtual Knowledge World for Natural Resource Management. In: Landscape Analysis and Visualisation: Spatial Models for Natural Resource Management and Planning, (Eds. Pettit, C., Cartwright, W., Bishop, I., Lowell, K., Pullar, D. and Duncan, D.), Springer, Berlin, pp 533-550.

Pettit, C. J., Sheth, F., Harvey, W., and Cox, M. 2009. Building a 3D Object Library for Visualising Landscape Futures. $18^{\text {th }}$ World IMACS / MODSIM Congress Proceedings, Cairns, 2009, pp.2244-2250.

Prusinkiewicz, P. 2004. Modelling Plant Growth and Development. Current Opinion in Plant Biology, 7, pp. 79-83.

Prusinkiewicz, P., and Lindenmayer, A. 2004. The Algorithmic Beauty of Plants (revised edition). Springer-Verlag, New-York.

de Reffye, P., and Hu, B.G. 2003. Relevant Qualitative and Quantitative Choices for Building and Efficient Dynamic Plant Growth Model: GreenLab Case. PMA03 Proceedings, Beijing, Oct 13-16, 2003, pp. 87-107.

Sarlikioti, V., Visser, P.H.B., and Marcelis, L.F.M. 2010. Exploring the spatial distribution of light interception and photosynthesis of canopies by means of a functional-structural plant model. Annals of Botany, 107(5), pp. 875-883

de Visser, P.H.B., Marcelis, L.F.M., van der Heijden, G.W.A.M., Vos, J., Struik, P.C., and Evers, J.B. 2002. 3D modelling of plants: a review. Report of the Virtual Plant Network Wageningen. Technical Report, Plant Research International B.V., University of Wageningen.

Vos, J., Evers, J.B., Buck-Sorlin, G.H., Andrieu, B., Chelle, M., and Visser, P.H.B. 2010. Functional-structural plant modelling: a new versatile tool in crop science. Journal of Experimental Botany, 61(8), pp. 2101-2115. 